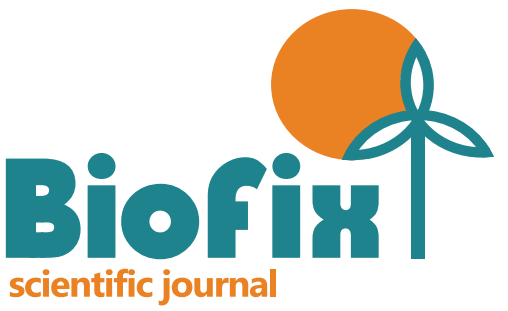

v. 2 n. Especial I MECFOR 2017

\section{MODELAGEM DA BIOMASSA RESIDUAL DA COLHEITA DE MADEIRA EM POVOAMENTOS SUBMETIDOS AO DESBASTE COM HARVESTER}

\author{
MODELING OF RESIDUAL BIOMASS OF WOOD HARVEST IN STANDS
} UNDER THINNING WITH HARVESTER
Recebido em 05/11/2017

Aceito em 10/11/2017

Publicado em 22/11/2017

DOI: dx.doi.org/10.5380/biofix.v2i0.56337

\author{
Carla Krulikowski Rodrigues ${ }^{1}$ \\ Eduardo da Silva Lopes ${ }^{2}$ \\ Afonso Figueiredo Filho ${ }^{3}$ \\ Dimas Agostinho da Silva ${ }^{4}$
}

Universidade Estadual do Centro-Oeste, Irati, Paraná, Brasil carlakr@gmail.com ${ }^{1}$,eslopes@unicentro.br² \&afigfilho@gmail.com ${ }^{3}$,

Universidade Federal do Paraná, Curitiba, Paraná, Brasil dimas.agostinho.silva@gmail.com ${ }^{4}$

\section{RESUMO}

Objetivou-se neste estudo ajustar modelos matemáticos para a estimativa da biomassa residual da colheita de madeira em povoamentos submetidos ao desbaste utilizando dados mensurados pelo harvester. O estudo foi realizado em uma empresa florestal localizada no município de Quedas do Iguaçu, Paraná. Os povoamentos avaliados foram de Pinus taeda L. submetidos ao desbaste nas idades de 7, 8, 9 e 10 anos. Através de um inventário florestal, cubagem com a simulação das medidas obtidas pelo cabeçote harvester e obtenção da biomassa seca dos resíduos, ou seja, acículas, casca, galhos e ponteiras, ajustou-se modelos de biomassa seca dos componentes das árvores presentes na biomassa residual. Por meio do método Stepwise, realizou-se a seleção das variáveis: idade do povoamento (I), diâmetro a 1,3 m do solo (DAP), altura comercial (AC) e volume comercial (VC). Em seguida, calculou-se o coeficiente de determinação ajustado e erro padrão da estimativa em porcentagem. $\mathrm{O}$ modelo de biomassa total de resíduos, ajustado com a seleção das variáveis DAP e VC, foi o que apresentou melhores parâmetros estatísticos, com coeficiente de determinação ajustado de 0,85 e erro padrão da estimativa de $26,90 \%$. Tal resultado comprova que o desenvolvimento desses modelos pode se tornar uma ferramenta inovadora para prever a biomassa residual existente ao nível operacional, para que possa ser inserida no hardware da máquina e aumentar a eficiência no planejamento do sistema de cavaqueamento e logística dos resíduos destinados à energia.

PALAVRAS-CHAVE: Bioenergia, Planejamento, Mensuração Florestal.

\section{ABSTRACT}

The aim of this study was to fit mathematical models to estimate residual biomass of the wood harvest in stands under thinning using data measured by harvester machine. The study was carried out in a forest company located in the municipality of Quedas do Iguaçu, Paraná State. Pinus taeda L. stands under thinning were evaluated at the ages of $7,8,9$, and 10 years. Through a forest inventory, tree scaling with the simulation of measurements by harvester head, and dry biomass of the residues: beads, bark, branches, and tips, biomass models of the tree components in the residual biomass were fitted. By means of the Stepwise method, the following variables were selected: stand age (I), diameter at $1.3 \mathrm{~m}$ (DAP), commercial height (AC), and commercial volume (VC). Then, the adjusted coefficient of determination and standard error of the estimate in percentage were calculated. The results showed the total biomass of residues, with the variables DAP and VC, was the fitted model that presented best statistical parameters, with adjusted coefficient of determination of 0.85 and standard error of the estimate of $26.90 \%$. This result proves that the development of these models can become an innovative tool to predict the present residual biomass at the operational level, so that it can be inserted into the machine hardware and increase the efficiency on the system planning of the wood chipper and the logistic of the residuals destined for the energy.

KEYWORDS: Bioenergy, Planning, Forest Measurement. 


\section{INTRODUÇÃO}

A necessidade de diversificação da matriz energética e as questões ambientais ligadas às emissões de gases de efeito estufa têm colocado a biomassa florestal em destaque nos últimos anos (BEN, 2017). O uso da biomassa residual da colheita de madeira para a geração de energia renovável é realizado por muitas empresas florestais no Brasil (IBÁ, 2017), entretanto, limitam-se ao corte raso e ao sistema de árvores inteiras. Isso se deve à falta de conhecimento da quantidade de biomassa produzida, visto que não é possível realizar relatórios da sua produção pelas máquinas florestais.

A estimativa de biomassa florestal é usualmente realizada por meio de metodologias baseadas em dados de inventário florestal, empregando-se fatores ou equações de biomassa, onde as variáveis independentes são os dados de diâmetro à altura do peito, altura total e volume individual (SOMOGYI et al., 2007). Geralmente, são realizadas amostragens destrutivas para a estimativa da biomassa, por meio da separação de seus componentes, que podem variar de acordo com os objetivos a serem alcançados (SILVEIRA et al., 2008).

Uma forma de estimar a biomassa florestal pode ser por meio dos dados mensurados pelo cabeçote harvester, pois, esse realiza medições de diâmetros e comprimentos das toras e calcula o volume de seções no momento do processamento da madeira. Esses dados podem ser utilizados como variáveis independentes nos modelos para estimativa da biomassa residual, devido a sua fácil obtenção por meio de um relatório disponibilizado no computador de bordo da máquina. Segundo KWF (2013), o processo de medição de diâmetros e comprimentos das toras pelo harvester ocorre pela contagem do número de giros realizados pelos rolos durante o processamento da madeira, enquanto o sistema de medição de diâmetro está ligado ao sistema mecânico e hidráulico das facas de desgalhamento ou rolos de alimentação do cabeçote.

Para Palander et al. (2009), Vesa e Palander (2010) e Ovaskainen et al. (2011), os modelos para estimar a biomassa residual produzida nas operações de colheita da madeira, por meio de dados mensurados pelo cabeçote harvester, podem ser inseridos no hardware da máquina e gerar informações de produção desses coprodutos. Entretanto, tais modelos são muito específicos quanto a espécie, idade, densidade de plantio, entre outras características dos povoamentos. Portanto, os gestores florestais devem utilizar os modelos em áreas com características semelhantes.

Neste estudo, objetivou-se avaliar o potencial de modelos para estimar a produção de biomassa residual da colheita de madeira em nível operacional de desbaste em Pinus taeda L. Assim, gera-se um instrumento auxiliar para o planejamento das operações de cavaqueamento e logística desse material com o uso dos dados do harvester, tais como idade do povoamento, diâmetro à altura do peito, altura e volume comercial, como fonte de informações de variáveis independentes que possam ser aplicados ao modelo de biomassa seca de resíduos.

\section{MATERIAL E MÉTODOS}

O estudo foi realizado em uma empresa florestal, localizada no município de Quedas do Iguaçu, estado do Paraná, Brasil, entre as coordenadas geográficas $25^{\circ} 26^{\prime} 27^{\prime \prime}$ S e $52^{\circ} 55^{\prime} 17^{\prime \prime}$ W. O clima da região é classificado como Cfa (Köppen). No local do estudo, predominam-se os solos do tipo Neossolo litólico, com relevo suavemente ondulado, declividade média de $8 \%$ e altitude média de $566 \mathrm{~m}$.

Os povoamentos estudados foram de Pinus taeda com 7, 8, 9 e 10 anos, de mesma capacidade produtiva e situados em sítios com as mesmas condições de solo e relevo. As características gerais dos povoamentos avaliados estão descritas na Tabela 1.

Tabela 1. Características gerais dos povoamentos de Pinus taeda

\begin{tabular}{lcccc}
\hline \multirow{2}{*}{\multicolumn{1}{c}{ Informações }} & \multicolumn{4}{c}{$\begin{array}{c}\text { Idade dos povoamentos } \\
\text { (anos) }\end{array}$} \\
\cline { 2 - 5 } & $\mathbf{7}$ & $\mathbf{8}$ & $\mathbf{9}$ & $\mathbf{1 0}$ \\
\hline Ano de plantio & 2008 & 2007 & 2006 & 2005 \\
\hline Poda baixa (Ano de execução) & 2012 & 2011 & 2010 & 2009 \\
\hline Árvores existentes em 2015 (ha) & 1.470 & 1.487 & 1.437 & 1.417 \\
\hline Taxa de mortalidade (\%) & 12,0 & 11,0 & 14,0 & 15,0 \\
\hline DAP médio (cm) & 18,1 & 18,3 & 19,6 & 19,1 \\
\hline h média (m) & 12,8 & 13,6 & 15,2 & 15,6 \\
\hline VMI (m ${ }^{3}$ ) & 0,160 & 0,173 & 0,225 & 0,215 \\
\hline
\end{tabular}

DAP = diâmetro à $1,3 \mathrm{~m}$ do solo; $\mathrm{h}=$ altura total $(\mathrm{m}) ; \mathrm{e} \mathrm{VMI}=$ volume individual das árvores $\left(\mathrm{m}^{3}\right)$.

Os desbastes aplicados foram classificados como misto, sendo caracterizados pelo corte sistemático da quinta linha, com remoção de $20 \%$ dos indivíduos, o que possibilitou a abertura das trilhas de operação das máquinas, em que a linha foi determinada pelo alcance máximo da grua do harvester (Figura 1). Foram também removidos $30 \%$ das árvores com características inferiores em duas linhas adjacentes às trilhas de tráfego, caracterizando como desbaste seletivo por baixo. 
(A)

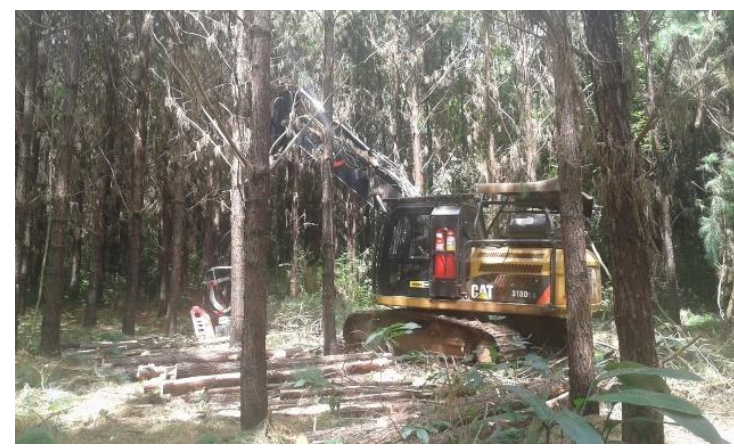

(B)

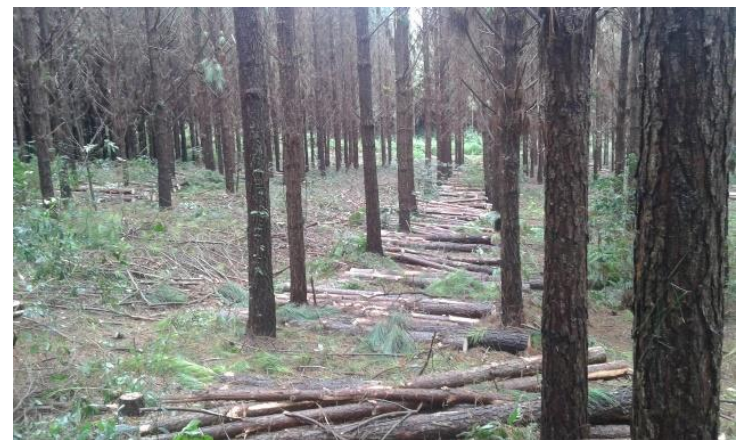

Figura 1. Harvester utilizado no desbaste (A) e aspecto do povoamento após a realização do desbaste (B).

Na execução dos desbastes, foram produzidos dois sortimentos de toras com casca, sendo classificados como toras grossas destinadas à serraria (diâmetro entre 15,0 e $35,5 \mathrm{~cm}$ e comprimento de $3,6 \mathrm{~m}$ ) e toras finas destinadas à energia (diâmetro entre 3,5 e 15,0 cm e comprimento de $3,05 \mathrm{~m}$ ). Enquanto a biomassa residual, composta pelos componentes: acículas, casca, galhos e ponteiras, permaneceu dispersa nas trilhas de tráfego das máquinas. As etapas das coletas dos dados foram realizadas conforme apresentadas na Figura 2.

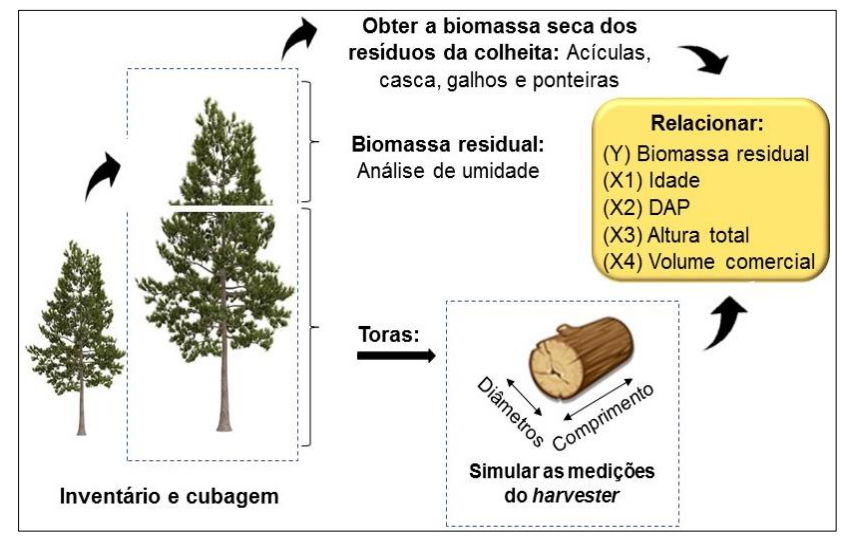

Figura 2. Fluxograma da coleta dos dados de inventário florestal, cubagem e biomassa seca nos povoamentos de Pinus taeda.

Inicialmente, realizou-se um inventário florestal e a seleção de árvores amostra nas quatro idades avaliadas, para a realização da cubagem e a quantificação da biomassa residual da colheita da madeira, sendo feitas em $17,9,10$ e 11 árvores amostras, respectivamente, nas idades 7, 8, 9 e 10 anos.

$\mathrm{Na}$ cubagem das árvores, foram mensurados os diâmetros à $1,3 \mathrm{~m}$ do solo, as alturas e os volumes comerciais, simulando as medições realizadas pelo cabeçote harvester. Enquanto na quantificação da biomassa verde, as árvores foram seccionadas em: toras grossas, quando existiam; toras finas; ponteira; acículas; casca; e galhos, sendo os quatro últimos componentes considerados biomassa residual e encaminhados para o laboratório para obtenção da biomassa seca.

$\mathrm{Na}$ análise de regressão, a massa dos resíduos florestais (Y), compostos pelas acículas, casca, galhos e ponteiras, foi considerada como variável dependente. Os galhos e ponteiras foram agrupados, uma vez que as ponteiras não apresentaram correlação com as variáveis independentes. Além disso, as variáveis independentes testadas para serem inseridas nos modelos foram: idade (I), diâmetro à $1,3 \mathrm{~m}$ do solo (DAP), altura comercial (AC) e volume comercial com casca (VC). Essas sofreram transformações e combinações para ajuste de modelos lineares múltiplos pelo método Stepwise, com auxílio do software SAS.

Os modelos ajustados foram apresentados com as seguintes estatísticas: coeficiente de determinação ajustado $\left(R^{2}{ }_{\text {aj. }}\right)$, erro-padrão da estimativa em porcentagem $\left(S_{y x} \%\right)$, valor de $F$, significância dos coeficientes de regressão $\left(\beta_{i}\right)$ e gráfico de resíduos.

\section{RESULTADOS E DISCUSSÃO}

Ao avaliar a biomassa residual da colheita de madeira em povoamentos de Pinus taeda submetidos ao primeiro desbaste, verificou-se que as maiores correlações ocorreram entre a biomassa total de resíduos por árvore (acículas, casca, galhos e ponteira) e as variáveis diâmetro à $1,3 \mathrm{~m}$ do solo (DAP) e volume comercial (VC) (Figura 3). Essa elevada correlação se refletiu no modelo ajustado para a biomassa total de resíduos.

$\mathrm{Na}$ Tabela 2 estão apresentados os modelos ajustados para a estimativa da biomassa seca dos componentes das árvores presentes na biomassa residual da colheita de madeira de Pinus taeda submetidos ao desbaste. Notou-se que, nos modelos ajustados para os componentes acículas, galhos e ponteiras, as variáveis DAP e/ou VC foram as selecionadas. Essas apresentaram os menores valores de coeficiente de determinação ajustado $\left(R^{2}{ }_{\text {j.j. }}\right)$, sendo 0,76 para os dois modelos, e maiores erros-padrão da estimativa em percentagem, com 51,99\% e 57,36\%, respectivamente. 

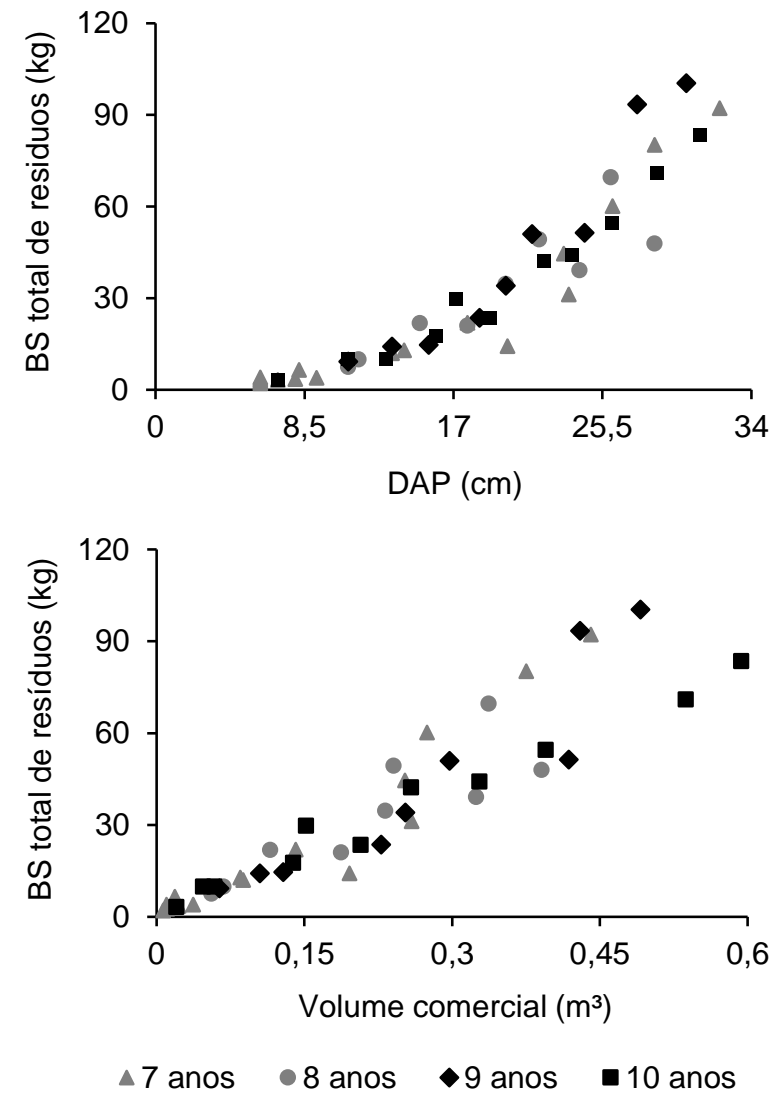

BS total de resíduos = biomassa seca da somatória dos componentes das árvores presentes na biomassa residual gerada na operação de colheita; e DAP = diâmetro à 1,3 m do solo.

Figura 3. Relação entre a biomassa seca total de resíduos com o DAP e o volume comercial dos povoamentos de Pinus taeda.

O melhor modelo ajustado encontra-se no componente total (soma dos componentes acículas, casca, galhos e ponteiras) de resíduos por árvore. Por meio das variáveis DAP e VC, os modelos apresentaram coeficiente de determinação ajustado de 0,85 , considerado elevado, e erro padrão de estimativa de $26,90 \%$, sendo aceitável para modelos de biomassa florestal. Entretanto, destaca-se que qualidade estatística do modelo para estimativa da biomassa seca do total de resíduos por árvore necessita ser melhorado, uma vez que a análise de resíduos do modelo mostrou que as estimativas são tendenciosas entre os diâmetros à 1,3 m (DAP) de 5 a $10 \mathrm{~cm}$, aproximadamente (Figura 4).

A maior tendenciosidade ocorreu nas primeiras classes de diâmetros dos povoamentos avaliados. Essas observações poderiam ser rejeitadas como outliers, entretanto, seriam eliminados os dados da primeira classe de DAP. Além disso, Vesa e Palander (2010) recomendam que tais dados sejam mantidos, para que futuras pesquisas estudem melhorias de amostragem. Operacionalmente, tal classe de DAP pode gerar erros amostrais, pois acarreta em falhas técnicas nos rolos de medição de comprimento das toras e diâmetros, devido à ausência de contado dos rolos com a madeira. Portanto, estudos em operações de corte raso em povoamentos com menor heterogeneidade da distribuição das classes de diâmetros podem apresentar melhorias em seus resultados.

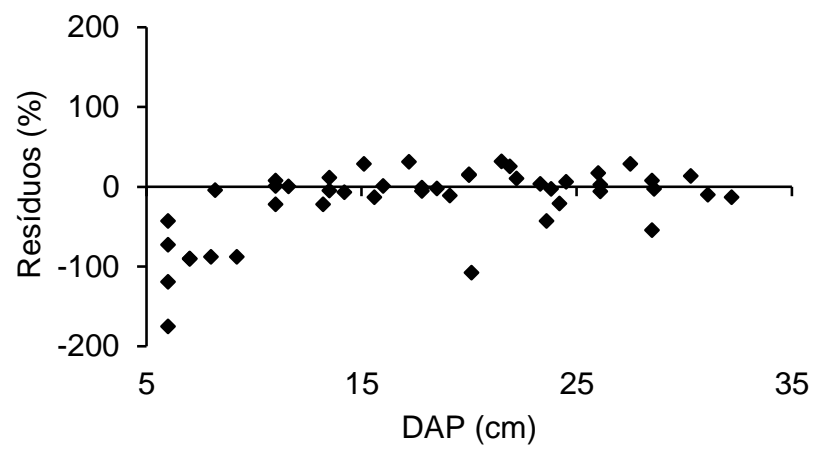

Figura 4. Distribuição dos resíduos do modelo de biomassa seca total para estimativa dos resíduos gerados na operação de desbaste de Pinus taeda.

Tabela 2. Modelos ajustados para a estimativa da biomassa seca dos componentes das árvores presentes na biomassa residual da colheita de madeira de Pinus taeda

\begin{tabular}{|c|c|c|c|c|}
\hline Componentes da árvore & Equações & $\mathbf{R}^{2}{ }_{\mathrm{aj}}$ & $S_{x y} \%$ & $\mathbf{F}$ \\
\hline Acículas & $\mathrm{BS}=0,4700^{\text {ns }}+0,0006^{* *} \cdot \mathrm{DAP}^{3}$ & 0,76 & 51,99 & $146,67^{*}$ \\
\hline Casca & $\mathrm{BS}=-0,0809^{\mathrm{ns}}+0,0205^{* *} \cdot \mathrm{DAP} \mathrm{P}^{2}-33,5213^{* *} \cdot \mathrm{VC}^{3}$ & 0,81 & 32,49 & $101,64 *$ \\
\hline Galhos + Ponteiras & $\mathrm{BS}=4,2838^{\text {ns }}+240,5050^{* *} \cdot \mathrm{VC}^{2}$ & 0,76 & 57,36 & $146,78^{*}$ \\
\hline Total de resíduo & BS $=5,1454^{*}+0,0030^{* *} \cdot D^{2} P^{3}-19,6292^{\text {ns }} \cdot V C^{3}$ & 0,85 & 26,90 & $133,63^{*}$ \\
\hline
\end{tabular}

$\mathrm{BS}=$ biomassa seca $(\mathrm{kg}) ; \mathrm{DAP}=$ diâmetro à $1,3 \mathrm{~m}$ do solo; $\mathrm{VC}=$ volume comercial; $\mathrm{R}^{2}{ }_{\text {aj. }}=$ coeficiente de determinação ajustado; $\mathrm{S}_{\mathrm{yx}} \%=$ erropadrão da estimativa em percentagem; $\mathrm{F}=$ valor do teste $\mathrm{F} ;{ }^{* *}=$ valores dos coeficientes significativos a $1 \%$, pelo teste $\mathrm{t} ;{ }^{*}=$ valores dos coeficientes significativos a $5 \%$, pelo teste $\mathrm{t}$; e ns = valores dos coeficientes não-significativos a $5 \%$, pelo teste $t$. 
De maneira geral, os resultados obtidos corroboram com a proposta de Vesa e Palander (2010) e Ovaskainen et al. (2011), os quais avaliaram o potencial de modelos para estimar a biomassa residual de tocos com os dados obtidos do computador de bordo da máquina de colheita de madeira, sendo o primeiro para abeto, pinus e bétulas, e o segundo apenas para abedo, ambos na Finlândia. Esses autores indicaram a possibilidade de utilizar dados mensurados pelo cabeçote harvester para estimar, em tempo real, a produção de biomassa residual da colheita da madeira, de forma a auxiliar no planejamento do uso dessa matéria-prima como fonte de energia renovável.

\section{CONCLUSÕES}

A biomassa residual da colheita de madeira pode ser estimada por meio de dados de diâmetro à 1,3 $\mathrm{m}$ do solo e volume comercial mensurados pelo cabeçote harvester, sendo o melhor modelo obtido para o total de resíduos por árvore.

O melhor modelo ajustado foi para a biomassa total, apresentando maior correlação com as variáveis independentes testadas e proporcionando melhores parâmetros estatísticos.

Os modelos ajustados para a estimativa da biomassa residual apresentam potencial para serem inseridos no software do computador de bordo do harvester e utilizados como ferramenta para obtenção de relatórios de produção desses produtos.

\section{REFERÊNCIAS}

BEN - BALANÇO ENERGÉTICO NACIONAL. Relatório síntese, ano base 2016. Rio de Janeiro: Empresa de Pesquisa Energética, 2017. Disponível em: <www.ben.epe.gov.br>. Acesso em: 23/10/2017.

IBÁ - INDUSTRIA BRASILEIRA DE ÁRVORES. Relatório anual da IBÁ 2017, ano base 2016. 77 p. Disponível em: <http://www.iba.org>. Acesso em: 18/10/2017.

KWF - Kuratorium für Waldarbeit und Forsttechnik. Lastenheft Harvestervermessung. Kuratorium für Waldarbeit und Forsttechnik e.V., Groß-Umstadt, 2013.

OVASKAINEN, H.; PIHLAJA, P.; PALANDER, T. Kantobiomassan määrän mallintaminen leimikoissa hakkuukonemittausten avulla. Metsätieteen aikakauskirja, n. 4, p. 290-293, 2011.

PALANDER, T.; VESA, L.; TOKOLA, T.; PIHLAJA, P.; OVASKAINEN, H. Modelling the stump biomass of stands for energy production using a harvester data management system. Biosystems Engineering, v. 102, n. 1, p. 69-74, 2009.

SILVEIRA, P.; KOEHLER, H. S.; SANQUETTA, C. R.; ARCE, J. E. O estado da arte na estimativa de biomassa e carbono em formações florestais. Floresta, v. 38, n. 1, p. 185-206, 2008.
SOMOGYI, Z.; CIENCIALA, E.; MÄKIPÄÄ, R.; MUUKKONEN, P.; LEHTONEN, A.; WEISS, P. Indirect methods of large forest biomass estimation. Europe Journal Forest Research, v. 126, n. 2, p. 197207, 2007.

VESA, L.; PALANDER, T. Modeling stump biomass of stands using harvester measurements for adaptive energy wood procurement systems. Energy, v. 35, n. 10, p. 3717-3721, 2010. 